\title{
-Review-
}

\section{A general concept of spiral volumetric CT and a newly developed, post-processing method designated as CT-endoscopy}

\author{
Hisashi Kobayashi \\ Department of Radiology, Nippon Medical School
}

Key words : spiral CT, 3D-CT, CT-endoscopy, virtual endoscopy, voxel transmission

In the last few years, the CT scanner has been greatly improved in terms of the output of X-ray units, storage capacity and processing speed. A spiral volumetric CT scanner with slip-ring system has made it easier to reform three-dimensional (3D) images ${ }^{1-14}$. Literature concerning the usefulness of 3D CT imaging itself in a clinical application is scarce. 3D-CT angiography ${ }^{15 \sim 17)}$ by intravenous injection of contrast media must play an important role in the clinical use of the 3D CT. However, with regard to vascular disorders such as an aortic dissection, the problem of the possible visualization of a false lumen without blood flow can not as yet be considered to be settled. Even in 3D-CT angiography the problem of observing the inner space of a vessel remains unsolved, and it remains as a dead space. We have developed a new CT-endoscopy (CTES) technique ('92), which is an attempt to solve an unsolved problem, in collaboration with HITACHI Medical Corp., Japan. In this article the author explains the general concept of spiral volumetric CT, and fully describes some initial clinical experiences with the CTES method.

\section{General concept of spiral volumetric CT}

\section{Spiral volumetric scan by the slip-ring mechanism of the scanner}

The volume scanner employs a highly reliable slip-ring mechanism in the power supply system, which is the heart of a continuous rotation CT scanner. The conventional CT scanner uses cables to supply electric power to the X-ray tube unit and other components, which requires repetitive reciprocating motions of rapid acceleration, braking and stopping. On the other hand, the volume scanner smoothly rotates only in one direction because the power is supplied through the slip-ring (Fig. 1). The slip-ring system does not require special maintenance such as preventive measures against high voltage discharge. Thus, stable high speed scans can be continuously performed over long periods.

\section{Volume scan mode and $3 D$ image reconstruction}

In this scanning mode the scanner is continuously rotated while the patient is moved on the tabletop at a constant speed, to image a wide anatomical region in the axial, longitudinal 


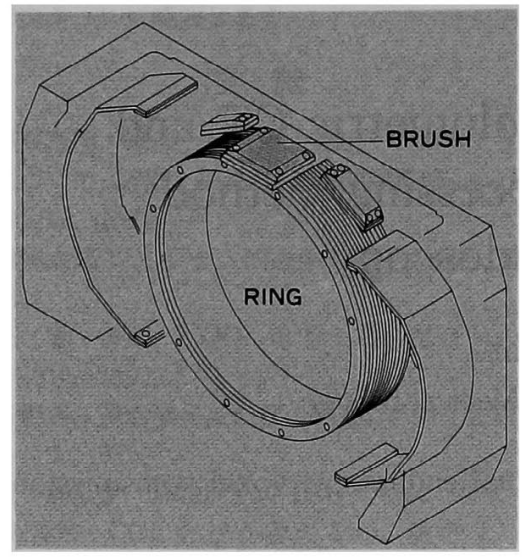

Fig. 1. Slip-ring mechanism. This slip-ring system, which is fundamentally different from the conventional scan mechanism, enables continuous rotation at a speed of one turn per second by the evolved scan mechanism and power supply system to the slip-ring, that is to say, "from cable to brush"

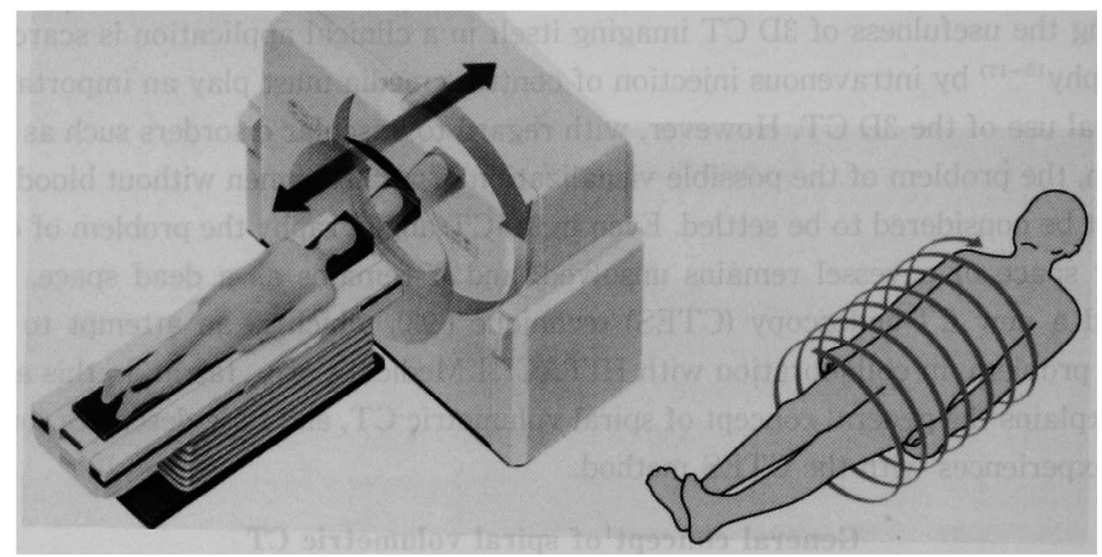

Fig. 2. Volume (i.e. spiral, helical) scan.

direction in a short time (Fig. 2). In conventional CT scanning, a brief stop is needed between scans and the tabletop is moved to the next scan position during each stop. In the volume scan mode, however, scan and tabletop increments are continuously made together with no loss of time. Therefore, it is possible to scan a wider region in a short time (Fig. 3). Scan throughput with the volume scan is about 5 times faster than that with the conventional scan. All data acquired are spirally contiguous to each other. Slice thickness can be freely selected, and the acquired data can be processed for an image reconstruction with desired slice pitches. Therefore, data measured with a single scan can be used to reconstruct a large number of CT images at any desired anatomical position and time phase in the flow of the scan. If these data are processed by the conventional method, the images reconstructed will possibly incur artifacts caused by table motion. For this volume scan (CT-W2000/3000), however, a unique method of interpolation is adopted to minimize such artifacts, thereby producing quality images comparable to those obtained through conventional image reconstruction. 


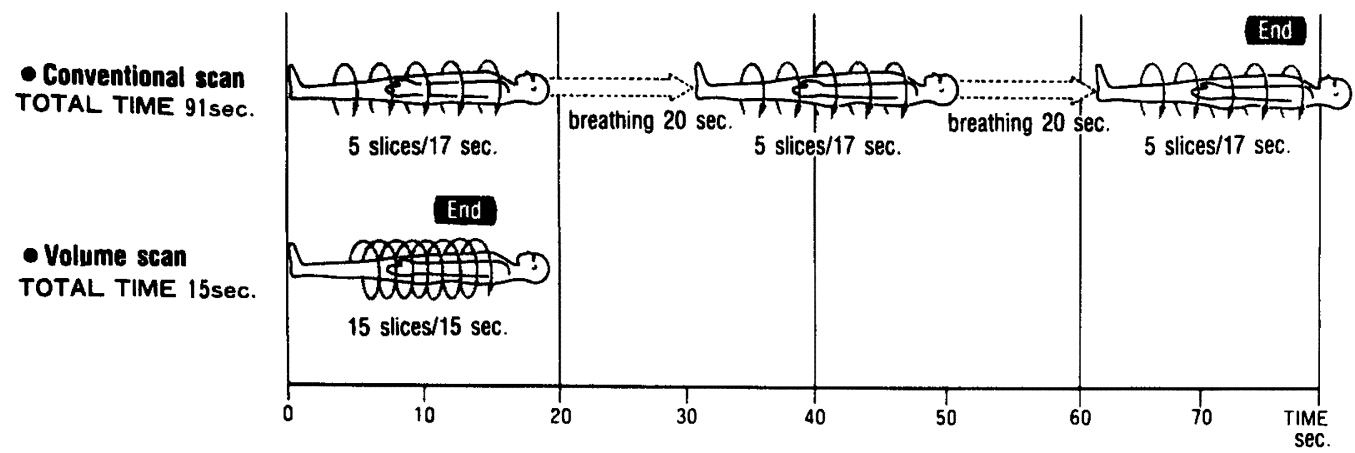

Fig. 3. Scanning program; the difference between conventional scan and volume scan. The volume scan is a scan mode to perform scan measurements and tabletop increments simultaneously without any loss of time, resulting in an efficient scan of a wide region in a short time.

For 3D image display, it is necessary to have many CT images of thin slices. However, the volume scan makes it possible to get a large number of images featuring an excellent contiguity between slices without the necessity of placing the patient under any spacial burden. Thus, 3D image display is possible with extremely high precision. Thanks to the short time feature of the volume scan, we can get a high precision 3D image display of thoracicoabdominal region and vascular system, which has not been possible with the conventional CT system.

\section{Voxel transmission method: A qualitative expression in $3 D C T$}

The author and colleagues have developed a new post-processing method which is a kind of volume-rendering projection method. It is, however, different from the conventional technique in many ways. The usual method of abstracting CT numbers for $3 \mathrm{D}$ reconstruction has been only to process them by a binary-calculating method at a threshold of the CT number. Therefore, it has been impossible to show a qualitative distinction among the different materials which exist within a threshold range: we can not distinguish calcification of the vascular wall from the contrast agent. While the maximum intensity projection (MIP) method makes it possible to distinguish between those two, it is conceivable that the MIP method sacrifices the biggest advantage of $3 \mathrm{D}$ manifestation, which is to heighten understanding of the positional relation of the intertwining structures. In a new method called "Voxel Transmission", all of the CT numbers of each voxel are precisely read, and they are reflected on a $3 \mathrm{D}$ coordinate axis with a gray-scale gradation. In a word, it may be possible to say that we have gained a unique expression of "quality" in 3D-CT images. The detailed formulae of voxel transmission method are omitted from this manuscript. The 3D-CT angiographic images obtained by the voxel transmission method are shown in figures 4 to 6 (Fig. 4〜6). 

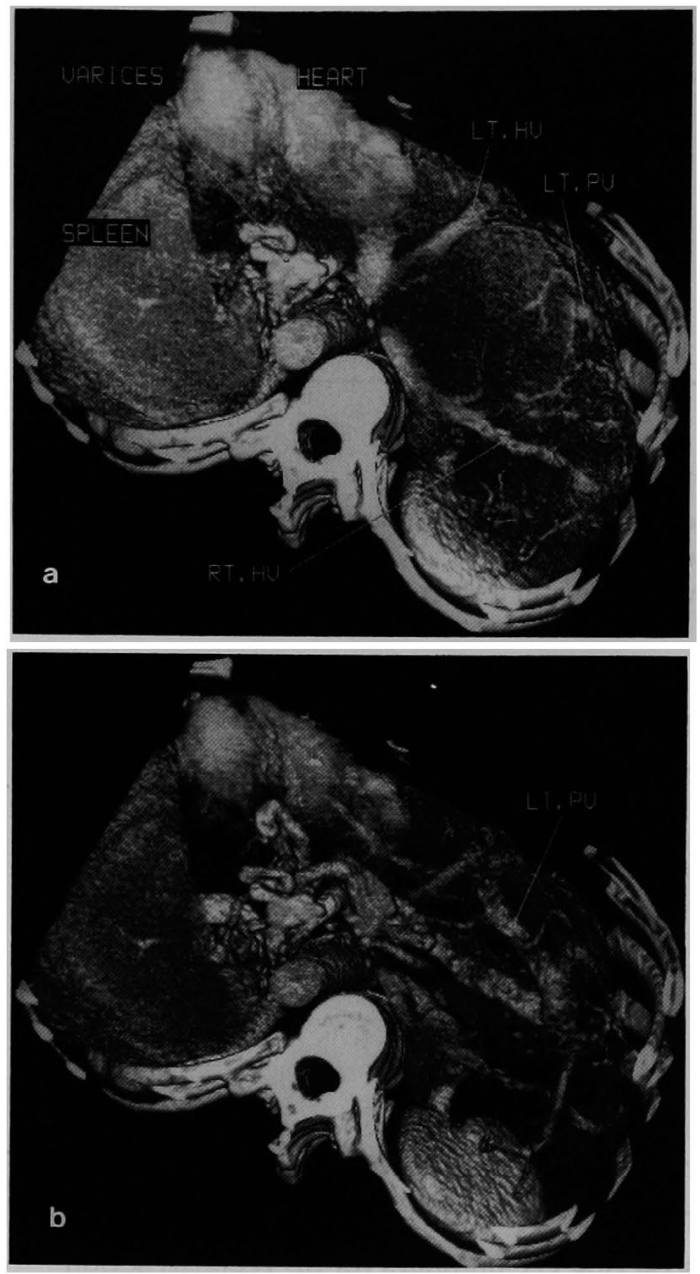

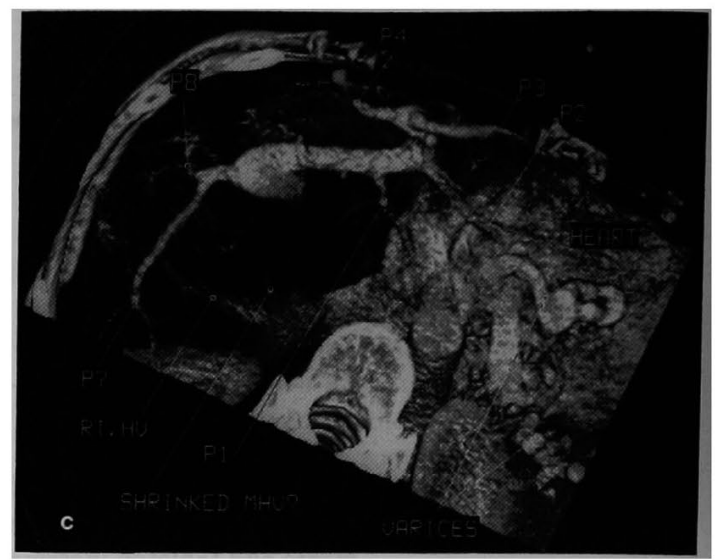

Fig. 4. Intravenous $3 \mathrm{D} \mathrm{CT}$ angiographic (CTA) images by the newly developed "voxel transmission" method. The patient has severe cirrhosis with portal hypertension. a) Top-down image of liver surface. Note a dilatation in the superficial veins surrounding the fibrotic scar. We can distinguish the vascular system from the parenchyma by the voxel transmission method. b) In the same angle of view, both the left portal vein and varices are visualized by manipulating the lower threshold of the CT number. c) Bottom-up view in the same patient. To heighten understanding of anatomical relations with the vascular system, 3D CTA will be useful.

\section{CT-endoscopy: Methodology and clinical evaluation}

\section{Patients and Methods}

To elucidate some changes on the inner surface of the tubular organs, one hundred and thirty-seven patients (examined in '92 and '93) with suspected cardiovascular disorders and other diseases of the tubular organs were reviewed in this series. In the 3D-CTES observation of the cardiovascular region, 32 aortic dissections, 9 aortitis and 6 intra-atrial thrombuses were evaluated with the use of the inner images. All of the 47 patients received a rapid intravenous injection of a non-ionic contrast medium $(300 \mathrm{mgl} / \mathrm{m} l)$ at 2 to $3 \mathrm{ml} / \mathrm{sec}$. via the antecubital vein. The total injected dose ranged from 96 to $140 \mathrm{~m} l$ (mean $121 \mathrm{~m} l$ )/body. Spiral scanning was started 25 to 30 seconds after the beginning of the injection of the contrast agent. Subsequently, serial scanning was done for 40 to 50 seconds at $5 \mathrm{~mm} / \mathrm{sec}$. in the table feed. All the CTES images were correlated with the conventional DSA images and/or the real time MPR, which was another manner of reconstruction by the same 


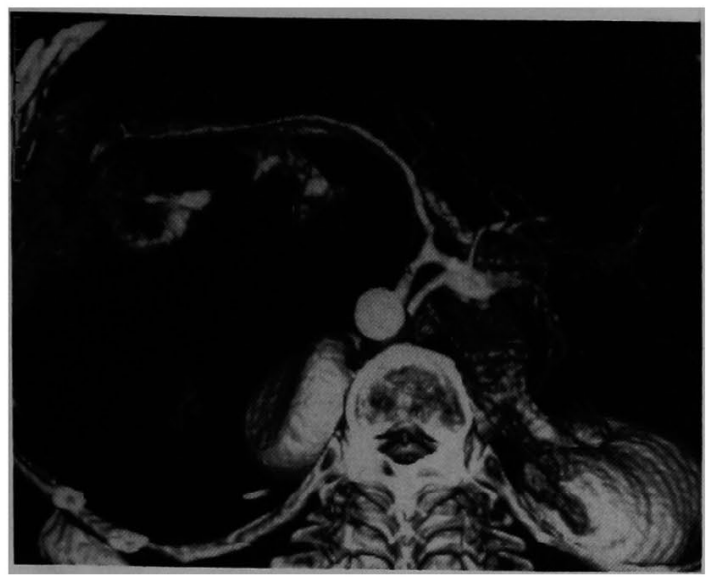

Fig. 5. Pay attention to the caudal pancreatic vessels which are displaced by the huge, avascular mass originating in the left adrenal gland. This image is intravenous angiography via the antecubital vein.

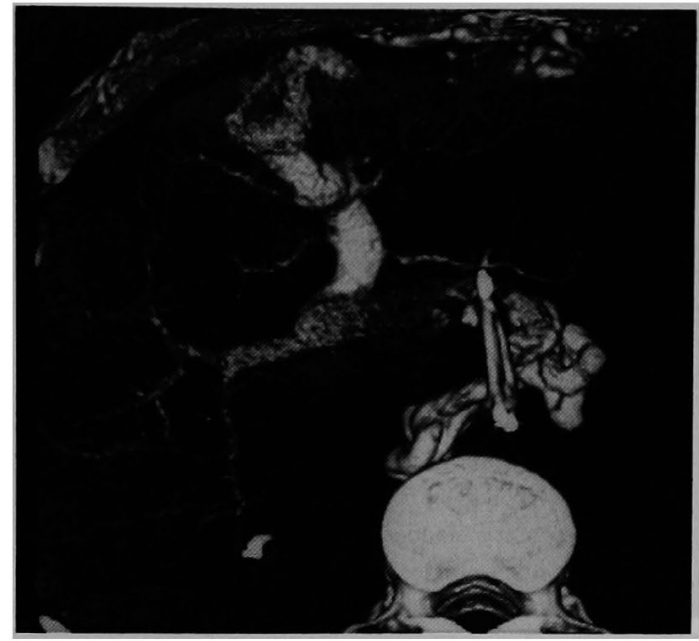

Fig. 6. 3D CTAP (CT during arterial portography) image clearly shows the dilatation of the paraumbilical vein and others.

scanning. Forty-one inspections of the common bile ducts (CBD), 18 cases of gallstones, 9 carcinomas of the CBD, 8 metastates from pancreatic carcinomas and 6 carcinomas from the gallbladder were included. Cholangiography was performed by the following three techniques: drip infusion method (DIC) with iotroxic acid $(50 \mathrm{mgl})$, percutaneous intrahepatic (PTC), and endoscopic retrograde cholangiography (ERC) in 20, 19 and 2 patients, respectively. Immediately after the cholangiography, spiral volumetric scanning was carried out, and $3 \mathrm{D}$ CTES images were then reconstructed and correlated with their conventional DIC, PTC and ERC on X-rayed films. Thirty-nine observations of the larynx, trachea and bronchus by the CTES technique were made without using any contrast media except the air in the airway. There were 25 pulmonary carcinomas, 7 laryngeal diseases, 4 mediastinal tumors and 3 traumatic injuries of the main bronchus. Broncho- and laryngo-fiberscopy was performed in 35 of 39 patients being observed by the CTES method. CTES of the urinary bladder was done in 10 patients including 7 patients with gynecological tumors adjacent to the bladder and 3 with bladder tumors. Cystography by spiral CT was done approximately 40 minutes after the intravenous injection of the contrast agent. The CTES images were reconfirmed by conventional cystoscopy with the use of optical fiberscopy, except in two cases, and by ultra sonography (US).

\section{Methodology of CT-endoscopic reconformation}

To reconstruct CTES images a newly developed volumetric scanner with a slip-ring system (CT W-2000/3000, Hitachi) was used. A 5 MHU output X-ray tube for scanning was controlled at conditions of $120 \mathrm{kV} / 200-300 \mathrm{~mA}$. The slice-width, speed of table-feed and pitch for $3 \mathrm{D}$ reconformation were set up at $5 \mathrm{~mm}, 5 \mathrm{~mm} / \mathrm{sec}$, and $2 \mathrm{~mm}$, respectively. The continuous scanning period was less than 50 seconds, and moreover, the scan-time per 
Table 1 Assessment of CT-endoscopy

\begin{tabular}{|c|c|c|c|c|}
\hline Region of observati & & No. of cases & Successes & Other confirmations \\
\hline $\begin{array}{l}\text { Aorta } \\
\text { dissection } \\
\text { aortitis }\end{array}$ & (41) & $\begin{array}{r}32 \\
9\end{array}$ & $\begin{array}{r}27 \\
9\end{array}$ & $\begin{array}{l}\text { MPR, DSA } \\
\text { MPR, DSA }\end{array}$ \\
\hline $\begin{array}{l}\text { Cardiac chamber } \\
\text { atria }\end{array}$ & (6) & 6 & 5 & US, axial CT \\
\hline $\begin{array}{l}\text { Common bile duct } \\
\text { cancer invasion } \\
\text { stone }\end{array}$ & (41) & $\begin{array}{l}23 \\
18\end{array}$ & $\begin{array}{l}16 \\
13\end{array}$ & $\begin{array}{l}\text { PTC, DIC, ERC } \\
\text { and/or MPR }\end{array}$ \\
\hline $\begin{array}{l}\text { Laryngobronchus } \\
\text { trachea \& bronchus } \\
\text { larynx }\end{array}$ & (39) & $\begin{array}{r}32 \\
7\end{array}$ & $\begin{array}{r}26 \\
7\end{array}$ & $\begin{array}{l}\text { bronchofiberscopy } \\
\text { laryngoscopy }\end{array}$ \\
\hline $\begin{array}{l}\text { Urinary bladder } \\
\text { gynecological tumor } \\
\text { bladder tumor }\end{array}$ & (10) & $\begin{array}{l}7 \\
3\end{array}$ & $\begin{array}{l}2 \\
2\end{array}$ & $\begin{array}{l}\text { cystoscopy, US } \\
\text { and/or MRI }\end{array}$ \\
\hline
\end{tabular}

rotation of the X-ray tube was one second. The 3D display was used to reconstruct threedimensional images from contiguous CT images. By applying Hitachi's volumetric scanner and our unique image processing method, which can extract $\mathrm{CT}$ number from the boundary region between a tubular organ and its lumen where X-ray absorption coefficients are different from each other, CTES has proved possible. This inner imaging is impossible by conventional 3D techniques. On 3D CTES reconstruction, the necessary condition was an apparent difference in $\mathrm{CT}$ number between the tubular walls and some component of the lumen. Therefore, in vascular imaging the injection of contrast material was necessary. In the trachea and bronchus it was also significant that there was air in the lumen: air is regarded as another type of contrast material. As regards the display methodology of 3D projection, CT W-2000 was applied with some voxel projecting method and the z-buffer gradient method. All 3D image processing was performed by the main console of the CT W$2000 / 3000$ itself without any network station.

\section{Clinical results}

The finished CT-endoscopic images in the 137 cases were checked by other diagnostic and CT processing methods, e.g. MPR (multiplanar reconstruction), axial image and cine mode, to confirm that the images had led to a correct diagnosis. The results are summarized in Table 1. The total number of successful observations in CTES amounted to 107 (78\%) of the 137 patients.

Cardiovascular region: In 41 (87\%) of the 47 observations by CTES, favorable results were obtained. CTES could clearly distinguish the false space from the true lumen in aortic dissection patients. Furthermore, it clarified the entry to false spaces, and showed intimal flaps, ulcer-like-projections and the blood stream in the false lumen, three-dimensionally (Fig. 7). One aortic dissection patient was followed up by CTES for one year. Observations demonstrated a slight enlargement of the entry, which proves the excellent reproducibility of the CTES imaging method (Fig. 8). In the 9 aortitis syndrome patients, it was possible to 

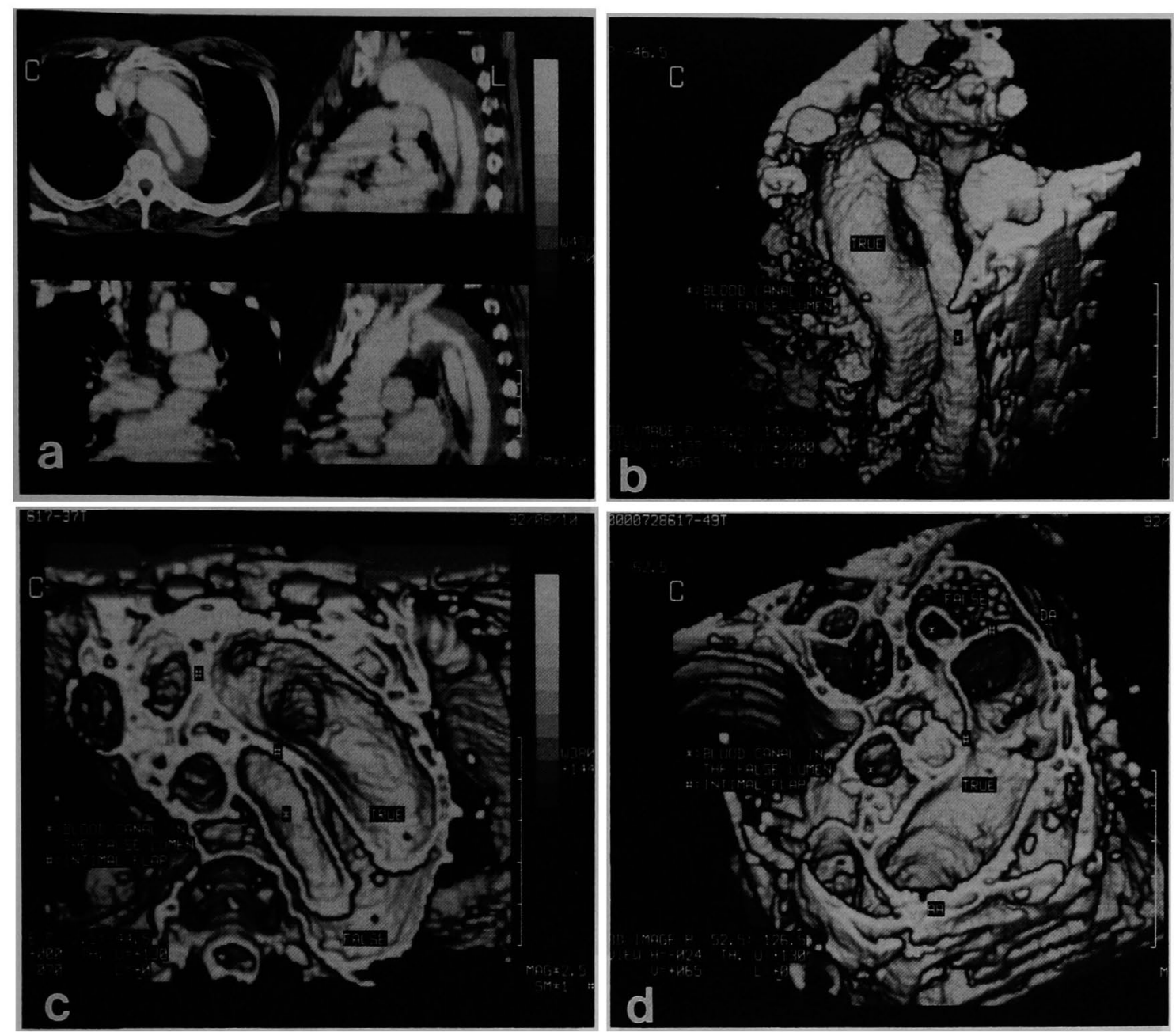

Fig. 7. a) A multi-planar image of severe dissection of the thoracic aorta. b) An intravenous 3D aortogram. Both the true lumen and the blood canal in the false space are visualized here. c) The inner image of the arch (bottom-up view) shows the detachment of the intima, blood canal in the false lumen and the first branching. d) Top-down view of the arch. These imaging techniques can clarify the status of the false lumen, which would not be clearly visualized by angiography.

judge whether there was any stenosis in the orifice, since the orifice of the first branch was depicted precisely, particularly in the arch of the aorta (Fig. 9). In one patient with aortitis who had a severe stenosis of the subclavian artery at a site just distal from the orifice, the CTES method revealed that the subclavian orifice was intact, and showed a remnant of the Botallo's duct, usually invisible in adults. In the cardiac chamber imaging both the atria were well-circumscribed, but the ventricles could not, of course, be visualized. The method succeeded in depicting the shape of the mural thrombus in the left atrium (Fig. 10). In a patient who had received an apicoaortic bypass operation, it was possible to peep through the side-to-end suture at the ascending aorta. 

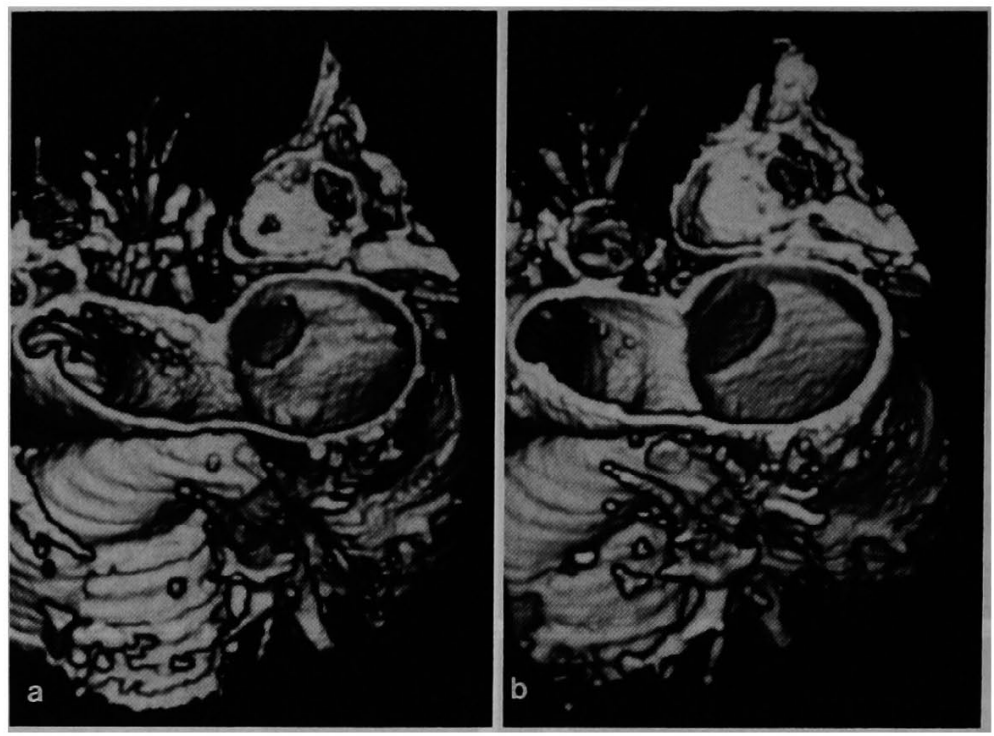

Fig. 8. A patient with aortic dissection was followed up by CT-endoscopy for one year. The entry existed in the proximal portion of the descending aorta; a) one year before. b) Present status of the lesion. This image demonstrates a slight enlargement of the entry.
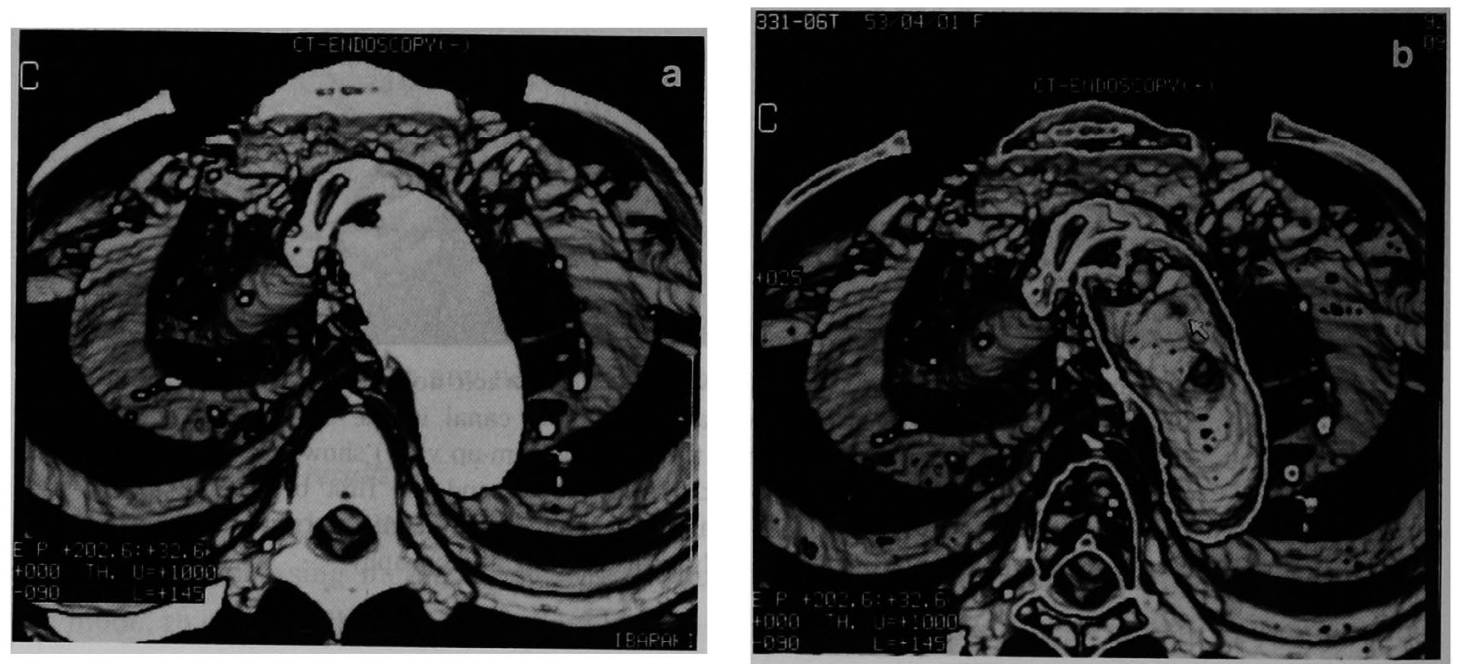

Fig. 9. A case with severe stenosis of the left common carotid artery due to aortitis syndrome. a) The arch is filled with contrast media, and no inner image is taken without CT-endoscopy technique. b) CT-endoscopy well depicts severe stenosis in the orifice of the common carotid artery.

The causes of unsuccessful CTES imaging in six cases were inadequate or mottled enhancement in the aorta, and the motion artifact caused by pulsation of the heart, which meant that there were lots of noises when the images were reproduced three-dimensionally.

Common bile duct (CBD): The inner image of the $\mathrm{CBD}$ was reconstructed from various kinds of cholangiography with CT scanning, as noted before. Successful images were 


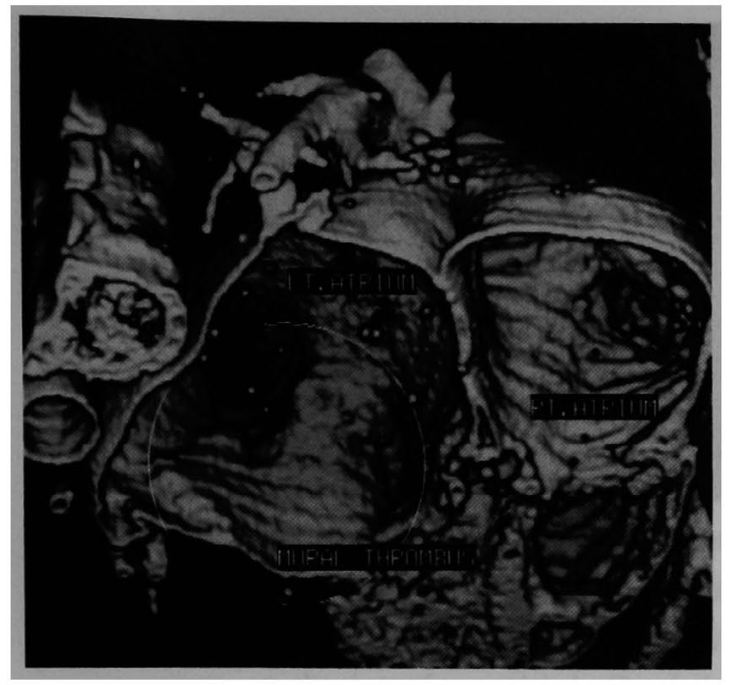

Fig. 10. An inner image of the atria. Mural thrombus is demonstrated in the left atrium of the heart (bottom-up view).

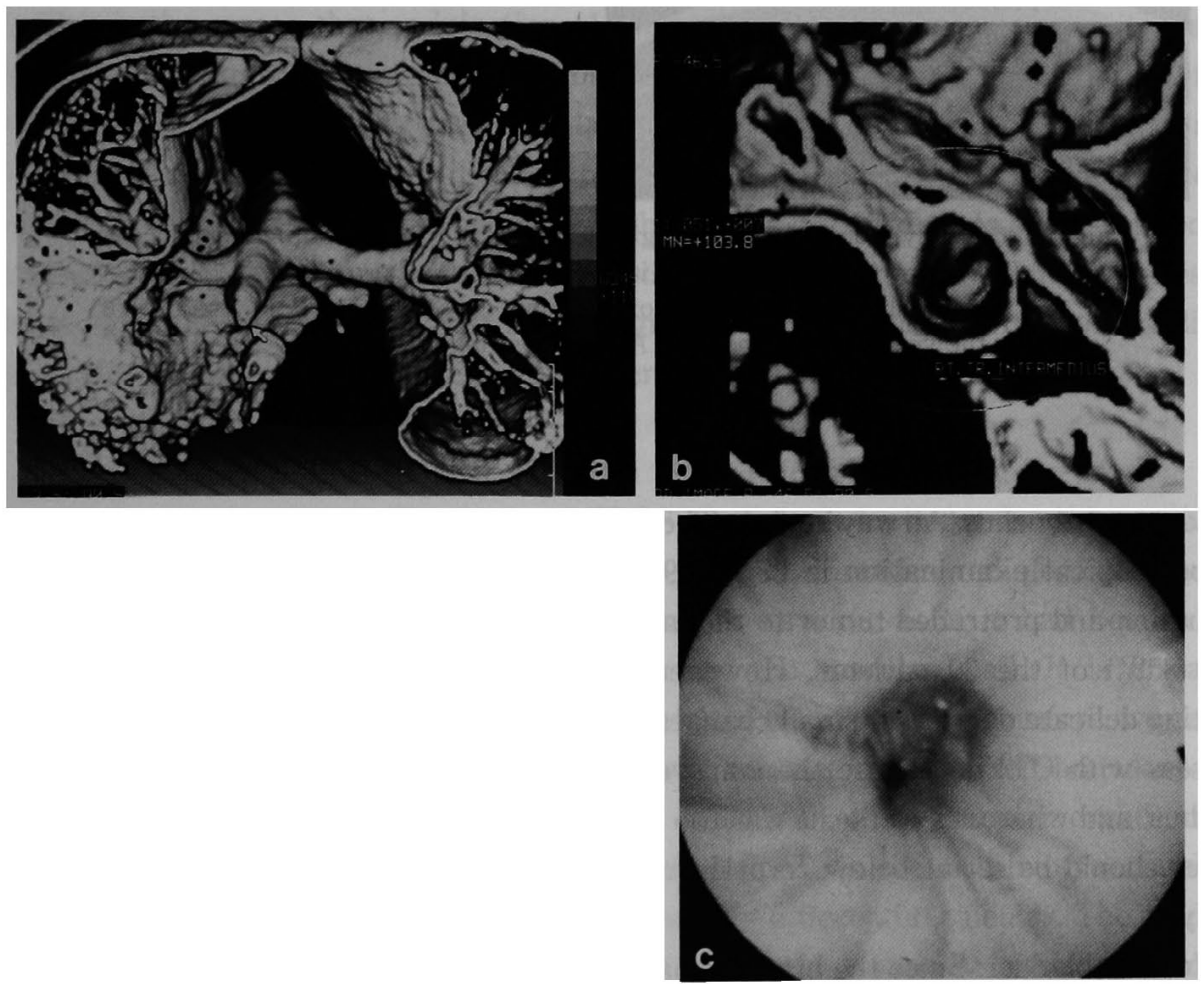

Fig. 11. Pulmonary carcinoma of the right hilum. a) The truncus intermedius is occluded, and secondary changes with atelectasis spread over the right inferior lung. b) Peeping into the right main bronchus with CT. endoscopy, we can clarify the linear obstruction. c) Bronchofiberscopy also shows the linear obstruction.

obtained from 29 (71\%) of 41 patients. However, the rest failed because the high level of contrast media in the $\mathrm{CBD}$ caused halation which radiated to the surrounding tissue of the 

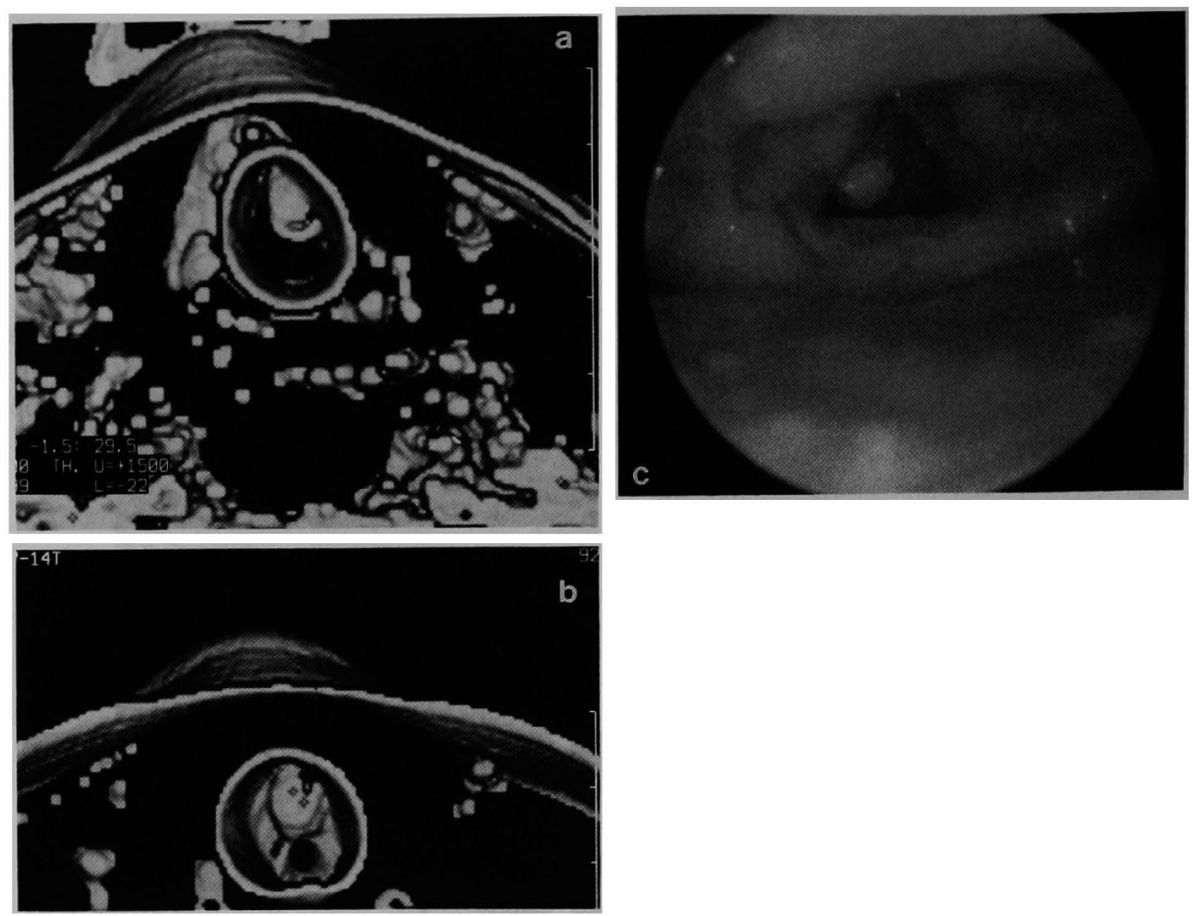

Fig. 12. A polyp located in the subglottic space. a) A bottom-up view indicates a polyp in the retrograde image against the laryngoscopic one. b) A small change in the shape of the polyp caused by phonation. Note that the vocal cords appear. c) A laryngoscopic view in the same case.

\section{CBD.}

Laryngobronchus/Airway region: The CTES images of this region were well confirmed by fiberscopical examination in 35 of 39 cases. The CTES findings, i.e, severe stenosis, obstruction and protruded tumor to the inside, correlated well with the fiberscopic findings in $33(85 \%)$ of the 39 patients. However, CTES was less successful than fiberscopy in detecting delicate degrees of small changes in the wall (Fig. 11, 12). Four cases were difficult to assess with CTES because the transverse portion reached out toward the upper lober bronchus, and what was worse, it was too narrow to reconstruct. The lower threshold of CT number should be set at below zero H.U. in order to express the boundary surface of the airway.

Urinary bladder: Since the bladders had not been fully filled with a contrast agent in the cystograms, we were unable to take successful CT images in six of the ten patients. In inner observations of the bladder contiguous to gynecological tumors, the results were unsatisfactory ( 2 of 7 cases). In one patient with a malignant bladder tumor whose contrast enhancement worked out well, the CTES image verified a stalk with the tumor in the lateral 3D display. 


\section{Discussion about CT-endoscopy}

Since the original outlines for this spiral volumetric CT scanning were prepared by Slavin ('69) ${ }^{18)}$, important advances have been made in various technological areas with CT. Spiral CT has spread throughout the world as a result of the technical innovations that have been made over the last twenty years. The introduction of spiral volumetric CT has made it easier to reconstruct 3D images. However, no one has been able to give concrete answers to questions concerning the clinical advantages of the 3D image itself. We initially reported the 3D CT-endoscopy (CTES) method ${ }^{19 \sim 21}$ as one of the clinical applications of the 3D image itself. As CTES images are analogous to those of fiberscopy, they may be able to express virtual fiberscopic images made from thin-slice data of the CT.

CT-endoscopy method; concerning "phantom wall" and "zero-crossing point": The CTES mode is a newly applied software which can extract the boundary lines between the walls of the tubular organs and inner components, viz, the blood, bile juices and the air in the lumen. Therefore, at the interface with both of those areas, a marked difference in CT number is needed. This is the reason that an injection of contrast media has been required. The air in the lumen is also regarded as a negative contrast material. As the steep gradient of CT number in an interface expresses itself as a large number of a differential coefficient in the first differential calculus, the "zero-crossing point" in the second differential is equal to the boundary point of the CT number. Therefore, both contiguous coordinates on the curved line must change into either plus or minus at the point, and thus, the boundary surfaces are depicted as the positive images against the background ${ }^{20}$. The Laplacian in applying digital imaging is understandable in the following, differential calculus:

$$
\begin{aligned}
\nabla f^{2}(i, j) & \equiv \Delta X^{2} f(i, j)+\Delta y f^{2}(j . j) \\
& =[f(i+1, j)+f(i-1, j)+f(i, j+1)+f(i, j-1)]-4 f(i, j)
\end{aligned}
$$

The CT W-2000/3000 has an automatic CTES mode built-in, but it is also possible to change to manual selection. When first looking at the CTES images, radiologists may find the "wall-like-thickness" of the tubular organs rather strange, as if it had been a real thickness of the vascular wall. However, this is not the vascular wall. It is in fact a non-existent imaginary thickness which exists only in the CTES mode. Therefore, we call its a "phantom wall". The phantom wall or thickness indicates a calculated boundary face between a surface of the contrast agent and the inner wall of the tubular organ, and is actually neither the contrast agent, nor the inner wall. It is the width of a couple of contiguous voxels at the zero-crossing point in the second differential calculus. The predominant feature of the CTES mode is that the determination of a boundary line is not made by the naked eye, but by a computer. Therefore, the reconstructed images have better reproducibility and objectivity. Besides, the CTES method makes it clear at a glance when a vascular lesion has a complicated stereostructure in the surrounding lumen.

Clinical applications of CTES: Cardiovascular region CTES images were successfully depicted in $87 \%$ of the patients with mural thrombus in the left atria, severe dissection and aortitis. Especially in the case of aortic dissection, clarifying the three-dimensional structure of the false space containing blood clots and a little residual flow was useful for surgeons in 
planning clinical management. Angiography could not have demonstrated the inner space of such a false lumen. 3D-CT angiography imaging reconstructed from volumetric data might present problems in the diagnosis of dissecting aneurysms, because the imaging expression of an intimal flap can be fabricated, whether the operator wants to or not, by manipulating the threshold ranges of the CT number. The distant range of intimal tears can not be measured precisely with the 3D-CT angiogram. Therefore, the measurement of dissections should be done with MPR or the sagittal sectional images of CTES. The other benefit of CTES is being able to observe the arch of the aorta. Angiofiberscopy has not usually been performed in observation of the arch. Even if it were possible to do, it would be difficult to wash out such a large amount of blood flow. CTES provided us with 3D inner information on the arch where the orifices of the first branches of the aorta must be observed. Observations of the common bile duct (CBD) were carried out with a $71 \%$ success rate. However, the inner images by CTES were inferior in diagnostic significance to the real-time MPR images. In laryngobronchial imaging with the CTES method, airways of over $7 \mathrm{~mm}$ in diameter were able to be reconstructed to the CTES images. In other words, the visible region ranged from the epiglottis to the truncus intermedius. As CTES can make an image in the opposite direction, that is, a retrograde view of the lumen, it was possible to depict the area beyond the severe stenosis where a fiberscope can not reach. In follow-up examinations during radiotherapy, the mediastinal bronchus invaded by hilar carcinoma was observed by the CTES technique. CTES of the urinary bladder was unsuccessful in 4 of 10 cases, because the bladder had not been filled sufficient contrast media, which caused strong halation.

From the above data and discussion it is possible to conclude that the CT-endoscopy method might be a safe and minimally invasive procedure to observe the inner surface of the tubular organs, particularly of the aorta, without any fiberscopic manipulation. Before closing, we would like to announce that a "cruising-eye view" method, which means a virtual endoscopy with an animated image passing through narrow arteries of $2 \mathrm{~mm}$ in size, has been developed by our group ${ }^{22,23)}$.

Acknowledgments: The author thanks K. Yamada and H. Ikoma (Central Hosp. of Ibaraki Pref.) for their enthusiastic support, and all the staff of the CT section at Hitachi Medical Corporation for their technological support. This work was supported in part by a grant from The Japanese Foundation for Research and Promotion of Endoscopy, '92, '93, and from The Public Welfare Foundation of NHK for Cancer Research, '92.

\section{References}

1) Napl, S.A., Marks M.P., Rubin, G.D., Dake, M.D., McDonnell, C.H., Song, S.M., Enzmann, D.R., and Jeffrey, R.B.: CT angiography with spiral CT and maximum intensity projection. Radiology, 185, 607 610, 1992.

2) Zimmerman, R.A., Gusnard, D.A., and Bilaniuk, L.T.: Pediatric craniocervical spiral CT. Neuroradiology, 34, 112-116, 1992.

3) Schwartz, R.B. Jones, K.M., Chernoff, D.M., Mukkerji,S.K., Khorasani, R., Tice,H.M., Kikinis, R., Hooton, S.M., Stieg, P.E., and Polak, J.F.: Common carotid artery bifurcation: Evaluation with spiral CT. (Work in progress) Rediology, 185, 513 519, 1992.

4) Tello, R., Scholz., E., Finn, J.P., and Costello, R.: Subclavian vein thrombosis detected with spiral CT and three-dimensional reconstruction. A.J.R., 160, 33〜34, 1993.

5) Costello, P. D., Ecker, C.P., Tello, R., and Horlnell, G.G.: Assessment of the thoracic aorta by spiral CT. A.J.R., 158, 1127 1130, 1992.

6) Remy-Jardin, M., Remy J, Wattinne L., and Giraud, F.: Central pulmonary thromboembolism; Diagnosis with spiral 
volumetric $\mathrm{CT}$ with the single-breath-hold technique; Comparison with pulmonary angiography. Radiology, 185, 381 387, 1992.

7) Suojanen, J.N., Mukherji, S.K., Dupuy, D.E., Takahashi, J.H., and Costello, P.: Spiral CT in evaluation of head and neck lesions. (Work in progress), Radiology, 183, 281 283, 1992.

8) Rigauts, H., Marchal, G., Baert, A.L., et al.: Initial experience with volume CT scanning. J. Comput. Assist. Tomogr., 14, 675 682, 1990.

9) Costello, P., Anderson, W., and Blum, D.: Pulmonary nodule: Evaluation with spiral volumetric CT. Radiology, 179, 875 876, 1991.

10) Bluemke, D.A., and Fishman, E.K.: Spiral CT arterial portography of the liver. Radiology, 186, 576 579, 1993.

11) Dupuy, D.E., Costello, P., and Ecker, C.P.: Spiral CT of the pancreas. Radiology, 183, 815 818, 1992.

12) Fishman, E.K., Wyatt, S.H., Ney, D.R., Kuhlman, J.E., and Siegelman, S.S.: Spiral CT of the pancreas with multiplanar display. A.J.R., 159, 1209 1215, 1992.

13) Ney, D.R., Fishman, E.K., Kawashima, A., Robertson, D.D., and Scott, W.W.: Comparison of helical and serial CT with regard to three-dimensional imaging of musculoskeletal anatomy. Radiology, 185, 865 869, 1992.

14) Kobayashi, H., Amemiya, R., Matsueda, K., et al.: Spatial tomography for detailed analyses of lung tumor; A preliminary report concerning analog-like processing images based on digital volumetric data. MEDIX, 24, 20 27, 1994.

15) Rubin, G.D., Dake, M.D., Napel, S.A., McDonnell, C.H., and Jeffrey, R.B.: Three-dimensional spiral CT angiography of the abdomen: Initial clinical experience. Radiology, 186, 147 152, 1993.

16) Matsueda, K., Kobayashi, H., Asato, Y., et al.: A new diagnostic imaging with a spiral volumetric CT; 3. 3D-CT angiography. (in Japanese) Innervision, 7(12), 40 45, 1992.

17) Kobayashi, H., Matsueda, K., Yoshimi, F., et al.: Topographic diagnosis of the abdominal tumor; Clinical advantages of 3D-CT images for a pre-surgical discussion. Japanisch-Deutsche Medizinische Berichte, 39, 124 136, 1994.

18) Slavin, P.E.: X-ray helical scanning means for displaying an image of an object within the body being scanned. US Patent No. 3432657, 1969.

19) Kobayashi, H., Okumura, T., Amemiya, R., and Hasegawa, H.: Works in progress: Endoscopic visualization of luminal organ and great vessels with three-dimensional CT scanner; A new trial using volumetric CT. (in Japanese) Nippon Acta Radiological, 52, 1195 1197, 1992.

20) Kobayashi, H., Matsueda, K., Asato, M., et al.: The development of a CT-endoscopy method. (in Japanese) Innervision, 8, 77 83, 1993.

21) Kobayashi, H., Okumura, T., Matsueda, K., Amemiya, R., and Hasegawa, H.: Inner images of the aorta; A new attempt with a volumetric CT sacenner. Radiology, 185(P), 364, 1992.

22) Hayashi, H., Kobayashi, H., and Kumazaki, T.: Virtual CT angioscopy cruising eye view; Development and clinical application. Work in progress (in Japanese), (in press) Nippon Acta Radiologica, 1996.

23) Hayashi, H., and Kobayashi, H.: Virtual CT endoscopy "Cruising-eye view"; theoretical background. New horizons of 30-CT imaging.-Current eoncepts and applications-(Ed. Kumazaki \& Kobayashi): P.12 16, Nankoudo, Tokyo, Japan, 1995.

(Received for publication, September, 2, 1994) 